University of Nebraska - Lincoln

DigitalCommons@University of Nebraska - Lincoln

Ralph Skomski Publications

Research Papers in Physics and Astronomy

June 1998

Finite-temperature behavior of anisotropic two-sublattice magnets

Ralph Skomski

University of Nebraska-Lincoln, rskomski2@unl.edu

Follow this and additional works at: https://digitalcommons.unl.edu/physicsskomski

Part of the Physics Commons

Skomski, Ralph, "Finite-temperature behavior of anisotropic two-sublattice magnets" (1998). Ralph Skomski Publications. 24.

https://digitalcommons.unl.edu/physicsskomski/24

This Article is brought to you for free and open access by the Research Papers in Physics and Astronomy at DigitalCommons@University of Nebraska - Lincoln. It has been accepted for inclusion in Ralph Skomski Publications by an authorized administrator of DigitalCommons@University of Nebraska - Lincoln. 


\title{
Finite-temperature behavior of anisotropic two-sublattice magnets
}

\author{
Ralph Skomski \\ Department of Physics and Astronomy, University of Nebraska, Lincoln, Nebraska 68588-0111
}

The finite-temperature magnetism of rare-earth transition-metal intermetallics is investigated by extending the $n$-component vector spin model to two-sublattice magnets. Mean-field analysis shows that the influence of the rare-earth anisotropy on the mean-field Curie temperature is much smaller than expected from the low-temperature rare-earth anisotropy. The use of ultraspherical polynomials yields a generalization of the famous $m(m+1) / 2$ power-law exponent to $m(m+n-2) /(n-1)$.

(C) 1998 American Institute of Physics. [S0021-8979(98)48811-X]

\section{INTRODUCTION}

Two-sublattice ferro- and ferrimagnets such as $\mathrm{Nd}_{2} \mathrm{Fe}_{14} \mathrm{~B}, \mathrm{Dy}_{3} \mathrm{Fe}_{5} \mathrm{O}_{12}$, and $\mathrm{SmCo}_{5}$ are physically interesting and technologically important materials. ${ }^{1-4}$ A particular feature of these materials is that their two-sublattice character is associated with nonequivalent crystallographic sites. ${ }^{5-7}$ This has to be contrasted to antiferromagnets having equivalent sublattices. ${ }^{8,9}$ For example, the square-lattice Ising antiferromagnet can be mapped onto the square-lattice Ising ferromagnet by simultaneously changing the sign of the exchange and reversing the spins of one sublattice. ${ }^{10}$

Rare-earth transition-metal compounds consist of transition-metal and rare-earth sublattices coupled by a comparatively weak intersublattice interaction. ${ }^{3,11}$ For the late iron-series elements the intersublattice exchange between rare-earth and transition-metal spins is antiferromagnetic ${ }^{11}$ so that, according to Hund's rules, light and heavy rare earths yield ferromagnetic and antiferromagnetic intersublattice coupling, respectively.

Atomic anisotropy energies are small, typically of order $E_{a} / k_{B} T \approx 1 \mathrm{~K}$, but due to interatomic exchange their influence on the magnetic properties is not restricted to low temperatures. Note that the theoretical description of the finitetemperature anisotropy of itinerant electrons is still in its initial stage, whereas the Heisenberg-type magnetism of localized electrons is comparatively well understood. Here we use a generalized $n$-vector model to investigate anisotropic two-sublattice magnets. Emphasis is put on two questions: (i) the effect the two-sublattice anisotropy on the Curie temperature, and (ii) the finite-temperature behavior of the net anisotropy.

\section{MODEL AND CALCULATION}

The $n$-component vector-spin or $n$-vector model (Fig. 1) is defined in terms of quasiclassical local magnetization vectors obeying $\mathbf{s}^{2}=s_{1}^{2}+\cdots+s_{n}^{2}=1$. $^{9,12}$ This definition includes the Ising model $\left(n=1\right.$, so that $\left.\mathbf{s}=s \mathbf{e}_{z}\right)$, the planar model $(n$ $\left.=2, \mathbf{s}=s_{y} \mathbf{e}_{y}+s_{z} \mathbf{e}_{z}\right)$, the classical Heisenberg model $(n=3$, $\left.\mathbf{s}=s_{x} \mathbf{e}_{x}+s_{y} \mathbf{e}_{y}+s_{z} \mathbf{e}_{z}\right)$, and the spherical model $(n=\infty)$. The $n=0$ model is known as the polymer model. ${ }^{13}$ The $n$-vector model is widely used to study finite-temperature magnetism. ${ }^{9,12}$ For example, the critical exponents of the spherical model are known exactly.
To generalize the $n$-vector model we have to introduce separate transition-metal and rare-earth sublattice magnetizations $\mathbf{S}$ and $\mathbf{S}$, respectively, so that $\mathbf{s}^{2}=1$ and $\mathbf{S}^{2}=1$. The spontaneous sublattice magnetizations are then given by $\mathbf{M}_{s T}=\mathbf{s} M_{0 T}$ and $\mathbf{M}_{s R}=\mathbf{S} M_{0 R}$, where the index 0 refers to the zero-temperature moment. Let us, for the moment, consider the mean-field Hamiltonian

$$
\begin{aligned}
\mathscr{H}= & -J_{T T} s_{z}\left\langle s_{z}\right\rangle-J_{R T} S_{z}\left\langle s_{z}\right\rangle-J_{T R} s_{z}\left\langle S_{z}\right\rangle \\
& -J_{R R} S_{z}\left\langle S_{z}\right\rangle-K_{T} s_{z}^{2}-K_{R} S_{z}^{2} .
\end{aligned}
$$

For the materials of interest, the transition-metal intrasublattice coupling $J_{T T}$ dominates the intersublattice exchange described by $J_{R T}$ and $J_{T R}$, whereas the rare-earth intrasublattice exchange $J_{R R}$ is negligibly small. $K_{T}$ and $K_{R}$ are the lowest-order uniaxial transition-metal and rare-earth sublattice anisotropy constants, respectively, and refer to the magnetic energy per atom. Due to the pronounced rare-earth spin-orbit coupling, $K_{R} \gg K_{T}$ for typical magnets. An exception are rare earths whose $4 f$ electron cloud is spherical, such as gadolinium. Note that putting $J_{R T}=J_{T R}=K_{R}=0$ and $K_{T}=K_{R}=0$ yields, for $n=3$, the well-investigated limits of the anisotropic one-sublattice ${ }^{14}$ and isotropic two-sublattice ${ }^{6,7}$ Heisenberg models, respectively.

The equilibrium behavior of the model Eq. (1) is given by the partition function

$$
Z=\int ' \exp \left(-\mathscr{H} / k_{B} T\right) d \mathbf{s} d \mathbf{S} .
$$

Here, the dash indicates that the conditions $\mathbf{s}^{2}=1$ and $\mathbf{S}^{2}$ $=1$ restrict the integration to the surfaces of $n$-dimensional spheres. The thermally averaged sublattice magnetizations
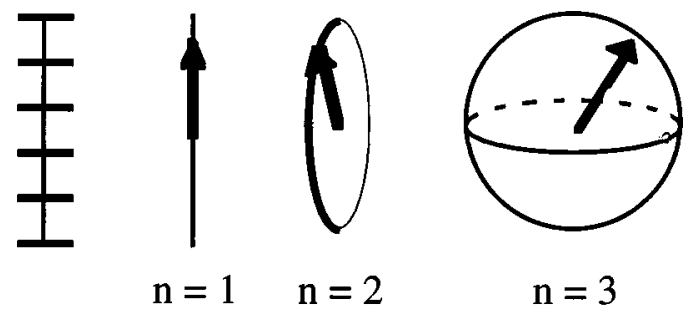

(a)

(b)

(c)

(d)

FIG. 1. Spin configurations: (a) $J=5 / 2$ Heisenberg model, (b) Ising model, (c) planar model, and (d) classical Heisenberg model. 


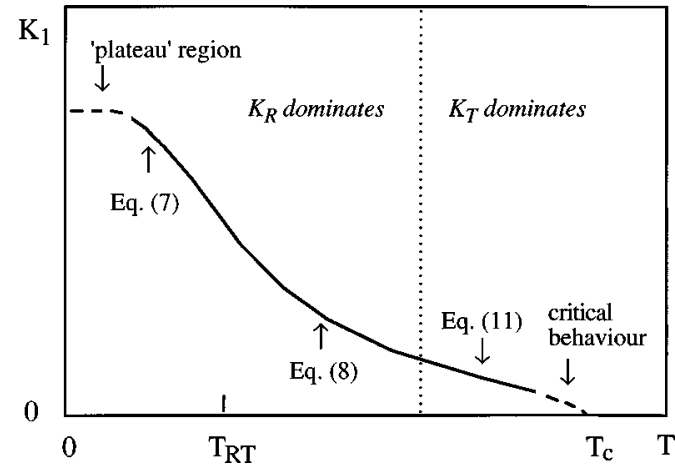

FIG. 2. Temperature dependence of magnetocrystalline anisotropy if both $K_{T}$ and $K_{R}$ are positive (schematic).

$M_{s T}\left\langle s_{z}\right\rangle$ and $M_{s R}\left\langle S_{z}\right\rangle$ and anisotropies $K_{T}\left\langle s_{z}^{2}\right\rangle$ and $K_{R}\left\langle S_{z}^{2}\right\rangle$ are obtained by direct integration or, more conveniently, by differentiating Eq. (2) with respect to $\left\langle s_{z}\right\rangle$ and $\left\langle S_{z}\right\rangle$. The resulting self-consistent equations are, in general, coupled and nonlinear. Mathematically, the calculation involves surface averages such as $\left\langle z^{m}\right\rangle_{0}=\int^{\prime} z^{m} d \mathbf{x} / \int d \mathbf{x}$. In particular, $\left\langle z^{2}\right\rangle_{0}=1 / n$ and $\left\langle z^{4}\right\rangle_{0}=3 / n(n+2)$.

\section{A. Curie temperature}

In the vicinity of the Curie temperature it is possible to expand $Z$ into powers of the small quantities $\left\langle s_{z}\right\rangle$ and $\left\langle S_{z}\right\rangle$. As in the case of isotropic two-sublattice magnets, ${ }^{6,7}$ the determination of the Curie temperature reduces to the calculation and diagonalization of a $2 \times 2$ secular matrix equation. In the present case,

$$
\left(\begin{array}{c}
\left\langle s_{z}\right\rangle \\
\left\langle S_{z}\right\rangle
\end{array}\right)=\left(\begin{array}{ll}
A_{T T} & A_{T R} \\
A_{R T} & A_{R R}
\end{array}\right)\left(\begin{array}{c}
\left\langle s_{z}\right\rangle \\
\left\langle S_{z}\right\rangle
\end{array}\right)
$$

where

$$
A_{i k}=\left(J_{i k} / n k_{B} T\right)\left\{1+\left[2(n-1) K_{i}\right] /\left[n(n+2) k_{B} T\right]\right\} .
$$

Neglecting $J_{R R}$ and taking into account the smallness of $J_{R T}, K_{T}$, and $K_{R}$, we obtain by eigenvalue analysis

$$
\begin{aligned}
T_{c}= & T_{T}+T_{R T}^{2} / T_{T}+\left\{2(n-1) /\left[n(n+2) k_{B}\right]\right\}\left(K_{T}\right. \\
& \left.+K_{R} T_{R T}^{2} / T_{T}^{2}\right),
\end{aligned}
$$

where $T_{T}=J_{T T} / n k_{B}$ and $T_{R T}=\sqrt{J_{R T} J_{T R} / n^{2} k_{B}^{2}}$.

The first two terms on the right-hand side of Eq. (5) are well known and describe the isotropic transition-metal and rare-earth contributions to the Curie temperature, respectively. ${ }^{3,7}$ The third term on the right-hand side of Eq. (5) is the anisotropy contribution to $T_{c}$. We see that the influence of the rare-earth anisotropy is smaller than expected from the value of $K_{R}$ by a factor $T_{R T}^{2} / T_{T}^{2}$, that is of order 0.05 for iron-rich rare-earth intermetallics.

Note that the anisotropy of the ideally anisotropic Ising model $(n=1)$ does not contribute to the Curie temperature, but from the leading term $T_{T}=J_{T T} / n k_{B}$ we see that the Ising Curie temperature is about three times larger than the Heisenberg Curie temperature.

\section{B. Anisotropy}

Figure 2 gives a schematic idea of the temperature dependence of the total anisotropy. Here we neglect the lowtemperature and critical limits (dashed lines) and focus on the intermediate regimes $T<T_{R T}$ and $T_{R T}<T<T_{c}$. In the classical Heisenberg model, the temperature dependence of $m$ th-order anisotropy contributions is proportional to the thermal average of the Legendre polynomials $\left\langle P_{m}\left(S_{z}\right)\right\rangle_{0}$, where the index 0 refers to the isotropic Hamiltonian. ${ }^{14}$ For the $n$-vector model we have to use ultraspherical polynomials $P_{m}^{(n)}(x) .{ }^{15}$ The first ultraspherical polynomials are $P_{0(n)}$ $=1, P_{1(n)}=x$,

$$
\begin{aligned}
& P_{2(n)}=\frac{1}{n-1}\left(n x^{2}-1\right), \\
& P_{3(n)}=\frac{1}{n-1}\left[(n+2) x^{3}-3 x\right],
\end{aligned}
$$

and

$$
P_{4(n)}=\frac{1}{n^{2}-1}\left[(n+2)(n+4) x^{4}-6(n+2) x^{2}-1\right] .
$$

For $n=2$, the ultraspherical polynomials are also known as Tchebicheff polynomials.

At low temperatures, the magnetization dependence of the Heisenberg anisotropy constants $K_{m / 2}$ is given by the famous power-law $K(T) / K(0)=\left(M_{s} / M_{0}\right)^{m(m+1) / 2}$ (Ref. 14). For example, $K_{1}(T) / K_{1}(0)=\left(M_{s} / M_{0}\right)^{3}$. Let us now generalize this power law to arbitrary spin dimensionalities, which is of some interest because statistical considerations often simplify in the limit of large spin dimensionalities. The starting point is the "low-temperature" expression $\int^{\prime} \exp (z / t) z^{m} d \mathbf{x}=1-m(n-1) t / 2$. By expressing the ultraspherical polynomials in terms of hypergeometric functions, ${ }^{15}$ we find after short calculation

$$
K_{1}(T) / K_{1}(0)=\left(M_{s} / M_{0}\right)^{m(m+n-2) /(n-1)} .
$$

Note that the exponent in this equation equals $m$ in the spherical model and $2 n /(n-1)$ for the lowest-order anisotropy constant $(m=2)$.

Equation (7) is restricted to one-sublattice magnets, but at very low temperatures $T<T_{R T}$ it remains valid if $M_{s}$ and $M_{0}$ refer to the rare-earth sublattice magnetization. In the practically important intermediate region $T_{R T}<T<T_{c}$, one has to consider the rare-earth sublattice in the exchange field of the transition-metal sublattice, ${ }^{3,17}$ which yields the approximate $1 / T^{A}$ power-law

$$
\frac{K_{R}(T)}{K_{R}(0)}=\frac{J_{R T}^{2}}{n(n+2) k_{B}^{2} T^{2}} .
$$

This prediction agrees fairly well with numerical studies on a quantum-mechanical single-ion mode ${ }^{16}$ and the estimate $A$ $=1.7 \pm 0.4$ deduced from literature data on $\mathrm{Sm}_{2} \mathrm{Fe}_{17} \mathrm{~N}_{x}$ and $\mathrm{Sm}_{2} \mathrm{Fe}_{17} \mathrm{C}_{x} \cdot{ }^{4}$

\section{Transition-metal sublattice}

The itinerant character of the $3 d$ electrons means that not only the spontaneous magnetization but also the mag- 
netic moment are temperature dependent. ${ }^{17-19}$ However, in most cases the thermal reduction of the magnetic moment does not exceed a few percent so that its neglect is a fair assumption. ${ }^{17-19}$ As a very crude approximation, we consider the mean-field Hamiltonian

$$
\mathscr{H}=-J_{T T} s_{z}\left\langle s_{z}\right\rangle-K_{T} s_{z}^{2}-U_{0} \mathbf{s}^{2},
$$

where $n=3$ and $\mathbf{s}^{2} \leqslant 1$ and the parameter $U_{0} \propto I-1 / D\left(E_{F}\right)$ is a Stoner-type single-site energy. ${ }^{19}$ Typically, $U_{0} \gg J \gg K_{T}$, so that in lowest-order $T_{c}=T_{T}\left(1+4 K_{T} / 15 k_{B} T_{T}-2 T_{T} / U_{0}\right)$. The reduction of $T_{c}$ is small in the region where Eq. (9) applies, but may nevertheless be larger than the $K_{1}$ contributions discussed in this paper.

In lowest order, the transition-metal anisotropy is given by the linear relation ${ }^{14}$

$$
K(T)=K(0)\left(1-T / T_{c}\right) .
$$

Note that this temperature dependence is even simpler than that of the spontaneous magnetization, which exhibits an implicit dependence on the Langevin function.

\section{DISCUSSION AND CONCLUSIONS}

The ultimate reason for the weak $K_{R}$ dependence in Eq. (5) is that the two sublattices are largely decoupled above $T_{R T}$. In the vicinity of $T_{c}$, the sublattice magnetizations are given by the eigenmode

$$
\left\langle m_{z}\right\rangle=\left\langle s_{z}\right\rangle+\frac{T_{R T}}{T_{c}}\left\langle S_{z}\right\rangle,
$$

showing that the mean-field magnetization near $T_{c}$ is dominated by the transition-metal sublattice. Of course, the meanfield approximation is incorrect in the vicinity of $T_{c} \cdot{ }^{10} \mathrm{How}-$ ever, the thermal sublattice decoupling is a mean-field effect starting far below $T_{c}$ and being most pronounced at high temperatures. As a consequence, even for $K_{T}=0$ the rareearth critical behavior is difficult to observe.

The key advantage of the $n$-vector model, namely its transparent physical meaning, is paid by an incorrect description of quantum-mechanical spin excitations. In particular, the classical $n$-vector model neglects the nonzero energy spacing between the quantum levels [Fig. 1(a)] and does not work very well at low temperatures (the "plateau" region in Fig. 2). ${ }^{16}$ Furthermore, Eq. (1) neglects exchange anisotropy $^{20}$ and magnetostatic dipole interactions. For this reason, our predictions are only semiquantitative.

In conclusion, we have analyzed the finite-temperature behavior of a generalized $n$-vector model. The influence of transition-metal and rare-earth anisotropies on the Curie temperature is treated in a mean-field approximation. Due to the weakness of the intersublattice coupling, the anisotropy contribution to the Curie temperature is determined by the transition-metal sublattice. The temperature dependence of the anisotropy may be approximated by a hierarchy of power laws.

\section{ACKNOWLEDGMENTS}

The author is indebted to J. M. D. Coey and J. Cullen for stimulating discussions.

${ }^{1}$ J. F. Herbst, Rev. Mod. Phys. 63, 819 (1991).

${ }^{2}$ K. N. R. Taylor and M. I. Darby, Physics of Rare-earth Solids (Chapman and Hall, London, 1972).

${ }^{3}$ Rare-earth Iron Permanent Magnets, edited by J. M. D. Coey (Oxford University Press, Oxford, 1996).

${ }^{4}$ R. Skomski and N. M. Dempsey, in Interstitial Intermetallic Alloys, edited by F. Grandjean et al. (Kluwer, Dordrecht, 1995), p. 653.

${ }^{5}$ L. Néel, Ann. Phys. (Paris) 3, 137 (1948).

${ }^{6}$ J. S. Smart, Effective Field Theories of Magnetism (Saunders, Philadelphia, 1966).

${ }^{7}$ A. Herpin, Théorie du Magnétisme (Institut National des Sciences et Techniques Nucléaires, Saclay, 1968).

${ }^{8}$ L. J. de Jongh and A. R. Miedema, Adv. Phys. 23, 1 (1975).

${ }^{9}$ D. C. Mattis, The Theory of Magnetism II (Springer, Berlin, 1985).

${ }^{10}$ L. P. Kadanoff, W. Götze, D. Hamblen, R. Hecht, E. A. S. Lewis, V. V. Palciauskas, M. Rayl, J. Swift, D. Aspnes, and J. Kane, Rev. Mod. Phys. 39, 395 (1967).

${ }^{11}$ I. A. Campbell, J. Phys. F 2, 147 (1972).

${ }^{12}$ K.-H. Fischer and A. J. Hertz, Spin Glasses (Cambridge University Press, Cambridge, 1991).

${ }^{13}$ P.-G. de Gennes, Scaling Concepts in Polymer Physics (Cornell University Press, Ithaca, 1979).

${ }^{14}$ R. Callen, J. Appl. Phys. 33, 832 (1962).

${ }^{15} \mathrm{H}$. Hochstadt, The Functions of Mathematical Physics (Wiley, New York, 1971).

${ }^{16}$ M. D. Kuz'min, Phys. Rev. B 46, 8219 (1992).

${ }^{17} \mathrm{P}$. Fulde, Electron Correlations in Molecules and Solids (Springer, Berlin, 1991).

${ }^{18}$ T. Moriya, Spin Fluctuations in Itinerant Electron Magnetism (Springer, Berlin, 1985).

${ }^{19}$ R. Skomski, J. Magn. Magn. Mater. 140-144, 2003 (1995).

${ }^{20}$ R. M. White, Quantum Theory of Magnetism (McGraw-Hill, New York, 1970). 